\begin{tabular}{|c|l|}
\hline Title & Morphol ogical investigations of disaccharide molecules for growth inhibition of ice crystals \\
\hline Author(s) & Uchida, Tsutomu; Nagay ama, Masafumi; Shibay ama, Tamaki; Gohara, Kazutoshi \\
\hline Citation & $\begin{array}{l}\text { Journal of Crystal Growth, 299(1), 125-135 } \\
\text { https://doi.org/10.1016/.jrysgro.2006.10.261 }\end{array}$ \\
\hline Issue Date & 2007-02-01 \\
\hline Doc URL & http://hdl.handle.net/2115/36102 \\
\hline Type & article (author version) \\
\hline File Information & uchida 5.pdf \\
\hline
\end{tabular}

Instructions for use 


\section{Morphological investigations of disaccharide molecules for growth inhibition of ice crystals}

Tsutomu Uchida*, Masafumi Nagayama, Tamaki Shibayama and Kazutoshi Gohara Division of Applied Physics, Graduate School of Engineering, Hokkaido University

*Author to whom correspondence should be addressed: N13 W8 Kita-ku, Sapporo 060-8628, Japan, Phone and Fax: +81-11-706-6635, E-mail: t-uchida@eng.hokudai.ac.jp 


\section{Abstract}

Freezing of solutions including disaccharides (trehalose, sucrose, and maltose) has been investigated by microscopic observations of freeze-fractured replicas using FE-TEM. Three typical features were observed: the smooth surface considered as the ice crystal, fine particles as the precipitated disaccharide molecules, and remaining part as the glass state of the solution. The expanded observations of fine-particle and its distribution investigations suggested that it was larger than $10 \mathrm{~nm}$ in size and averaged approximately 20-30 $\mathrm{nm}$ in diameter. The smallest particle was estimated to include several hundred disaccharide molecules. Based on systematic observations of trehalose solutions regarding concentrations and freezing rates, we concluded that ice crystal growth was inhibited by trehalose molecules. Since the ice crystal size reduced exponentially with increase in trehalose concentration, we could control ice crystal size formed in the frozen material. The growth inhibition of ice crystals with trehalose resulted both from a reduction in the free water in the solution due to a significant hydration effect and from an enhancement of nucleation of the ice crystals. It was confirmed that trehalose was more effective than the other disaccharide solutions examined for inhibiting the growth of ice crystals.

Key Words: A1. Nanostructures, A1. Solubilities, A2. Growth from solutions, B1. Biological substances, B1.

\section{Sucrose}

PACS code: 81.10 Dn Growth from solution 


\section{Introduction}

Numerous studies have been undertaken to preserve living bodies and foods from freezing by the addition of cryoprotecting materials including natural substances (e.g., sugars and proteins) and synthetic chemicals (e.g., glycerin and dimethyl sulfoxide). The effectiveness of such materials has been assessed for the freezing and freeze drying of cells; further, these materials have attracted attention from the viewpoint of developing a method for the physical fixation of samples examined using electron microscopy. It is considered that these materials specifically interact with a particular surface of ice crystals and thereby inhibit crystal growth; however, the mechanism of this inhibition is still largely unknown. Observation on a molecular scale is a promising approach for the clarification of this mechanism, although it is still difficult to perform the microscopic observation of water solutions.

Trehalose and sucrose, which consist of fructose and glucose rings connected by a glycosidic bond, are naturally occurring compounds found in cryoprotectants. Trehalose is primarily found in animals capable of enduring cold temperatures, whereas sucrose is typically found in plants [1]. These compounds are considered to play important roles in the cryoprotection of living cells, primarily via one or both of two mechanisms, i.e., they protect the lipid bilayer via the hydrogen bonding of sugar molecules and inhibit the growth of ice crystals in extracellular space. Based on the interactions between sugar molecules and the lipid bilayer, it has been suggested that the molecular mechanism underlying this cryoprotective effect is the hydrogen bonding of trehalose molecules to the bilayer head group [2]. A simulation of the interaction of the lipid bilayer with trehalose has revealed that only marginal changes occur in the lipid bilayer. 
On the other hand, the interaction between the sugar and water molecules has been investigated both by macroscopic observations (for example, [3-5] where the melting point of sugar solutions has been determined) and by microscopic observations (for example, [6-9] where the interaction has been measured via Raman or infra-red spectroscopy). Sei et al. [4, 5] found that the melting points of trehalose and sucrose solutions were lower than the molar freezing point depression. They explained this phenomenon by considering the ratio of the hydrated water around sugar molecules to that of free water, because these sugars are known to strongly interact with water. Therefore, the cryoprotective mechanism of sugar is considered to be controlled by the hydration of sugar molecules in solution. Raman spectroscopic observations have revealed that disaccharides promote a destructive effect on the tetrahedral hydrogen-bond network of pure water [9]. The results of such studies have led to the hypothesis that disaccharides obstruct the crystallization process, thereby destroying the network of water in a manner similar to that occurring in ice. This hypothesis qualitatively coincides with the conclusions suggested by the macroscopic observations.

Although the hydration structures of water in contact with disaccharides are likely to exhibit dimensions that would render them stable for electron microscopic observation, what appears to be the most relevant technique-freeze fracture-is not generally applicable, because it relies on the freezing process that by its very nature, changes the concentration of the solution and hence the hydration structure of the trehalose solution. However, it is known that aqueous solutions of certain types of polymers [10] and maltodextrin [11] can be quickly frozen to yield vitreous states under favorable conditions. Thus, the morphologies revealed by the electron microscopic examination are expected to correspond to the actual microstructures of these solutions. 
In the present study, we observed a freeze-fractured replica image of disaccharide (trehalose, sucrose, and maltose) solutions using a field-emission type transmission electron microscope (FE-TEM), and we investigated the relationship between disaccharide molecules and water (ice) in the frozen solutions. The TEM images indicated that numerous hydrated trehalose molecules precipitated to form uniformly-sized fine particles. Sucrose solutions provided similar images with trehalose, but a less inhibitory effect of sucrose on the growth of ice crystals was observed. Furthermore, maltose was found to be difficult to precipitate, and it marginally inhibited the ice crystal growth.

\section{Experimental}

We focused primarily on trehalose, which has been considered as a cryoprotective material that reduces the freezing rate of ice [5]. Research-grade trehalose dihydrate crystals were donated by Hayashibara Biochemical Laboratories, Inc. To compare the properties observed in the various trehalose solutions, we conducted similar investigations using sucrose (of the highest quality; Sigma-Aldrich Japan, Inc.) and maltose (research grade, Hayashibara Biochemical Laboratories, Inc.). The purity of the distilled and deionized water used for conductivity purposes was approximately $5 \times 10^{-6} \mathrm{~S} \mathrm{~m}^{-1}$.

We prepared the trehalose solutions at concentrations ranging from $3 \mathrm{wt} \%$ to $50 \mathrm{wt} \%$. The concentration of the solutions was determined by measuring the masses of the disaccharides and pure water taking into consideration the water of crystallization. The accuracy of the concentration was estimated to be $\pm 0.1 \mathrm{wt} \%$. Then, these solutions (approximately 20 $\mathrm{mm}^{3}$ ) were rapidly frozen in a liquid nitrogen atmosphere (approximately $80 \mathrm{~K}$ ). To avoid the bubbling of solution, the sample was kept just above the liquid nitrogen surface. Under these conditions, the freezing rate was approximately $3 \times 10^{2}$ 
$\mathrm{K} \mathrm{min}{ }^{-1}$. The order of this value was almost similar (ranging within $\pm 50 \mathrm{~K} \mathrm{~min}^{-1}$ ) in all trehalose solutions, even though the solution had a relatively wide concentration range. Then, the frozen sample was introduced into the freeze fracture replica sample preparation system (JEOL, type JFD-9010). The sample was fractured under a vacuum $\left(10^{-5}\right.$ Torr) and at a low temperature $(150-170 \mathrm{~K})$. A replica film of this fractured surface was prepared by evaporating platinum and carbon. To observe the replica samples, we used FE-TEM (JEOL, type JEM-2010) at an accelerating voltage of $200 \mathrm{kV}$. An imaging plate was used to record the observed images.

To investigate the effects of the freezing rate on the morphology of the respective samples, we also observed samples prepared at slow and rapid freezing rates. The slow freezing rate samples were prepared at a relatively high temperature of approximately $255 \mathrm{~K}$ (freezing rate: approximately $1 \mathrm{~K} \mathrm{~min}^{-1}$ ). The rapid freezing rate samples were prepared by introducing similar volumes of trehalose solution stored in a glass capillary (inner diameter: approximately $1 \mathrm{~mm}$ ) into liquid propane at a temperature of approximately $80 \mathrm{~K}$ (freezing rate: approximately $3 \times 10^{3} \mathrm{~K} \mathrm{~min}^{-1}$ ). The replica preparation processes for these samples were the same as those mentioned above.

\section{Results and Discussion}

\section{<TEM observations of quenched trehalose solutions $>$}

Figures 1a-1f show the TEM images of the freeze-fractured replica samples of trehalose solutions at various concentrations.

All the samples were quenched at a cooling rate of $3 \times 10^{2} \mathrm{~K} \mathrm{~min}^{-1}$. The bar in each picture is $100 \mathrm{~nm}$ in length, with the exception of some of the inner small pictures. A smooth surface area and spherical or oval-shaped fine particles (at sizes of 
several tens of nanometers in diameter) can be observed in each picture. The areas of the smooth surface were found to decrease with an increase in the trehalose concentration of the original solution, and the intermediate layers of these areas were filled with fine particles. Because the solution contained only pure water and trehalose, the smooth surface was considered to be the ice crystal, whereas the fine particles were considered to be trehalose molecules. The area around the fine particles that was different from the smooth surface was therefore considered as the glass phase of the solution.

The effects of the freezing rate on the relationship between the smooth area and another area containing fine particles were investigated using trehalose solutions (having $40 \mathrm{wt} \%$ and $35 \mathrm{wt} \%$ concentrations). The low freezing rate sample exhibited only smooth areas, but no fine particles (Fig. 2a) were observed. This result may be due to the removal of the entire solute from the large ice crystal during slow ice formation such that the concentrated solute was no longer within the area of observation. On the other hand, the TEM images of the rapid freezing rate sample revealed uniformly distributed fine particles in the intact areas of the sample (the sizes of which were similar to those observed in Fig. 1), as well as in the scant smooth surface area (Fig. 2b). This result may be interpreted as an incomplete separation of the solute from the solvent during the process of rapid freezing. These observations also support the interpretation that the smooth surface was the ice crystal and the fine particles represented trehalose molecules.

The ice crystals observed in samples at lower concentrations were so large that their precise shapes and numbers could not be determined (see Figs. 1a and b). However, the crystals became sufficiently small for TEM observation at higher trehalose concentrations. As shown in Figs.1c-1f, the ice crystals appeared to be oval elongated shapes and pointing in a certain direction. This elongation might have resulted from the growth of ice crystals along a preferential direction under the 
present freezing conditions. Further increases in the trehalose concentration led to a reduction in the size of the ice crystals and shortened the elongation; which showed a facet on the ice crystal. The distance between ice crystals also increased. These phenomena indicate both a reduction in the amount of $\mathrm{H}_{2} \mathrm{O}$ molecules available to form ice crystals and a reduction in the growth rate of the ice crystals. The increase in the trehalose concentration may also affect the degree of supercooling of the solution, which would control the diffusion rate of the heat formed in the ice crystal. Therefore, the growth rate was controlled either by the rate of supply of $\mathrm{H}_{2} \mathrm{O}$ molecules from the surrounding solution to the ice surface or by the diffusion rate of the heat formed from the reaction sites or by the kinetic inhibition of the ice crystal formation. If trehalose inhibits the ice crystal growth kinetically, we would find fine particles on the edge of a smooth area. However, the TEM images revealed that only a small amount of fine particles was located just along the boundary of the smooth area. Therefore, it appears that the main rate-determining process is the rate of supply of $\mathrm{H}_{2} \mathrm{O}$ molecules from the solution.

Figure 3 shows the distribution of ice crystals in terms of (a) size and (b) number concentration, depending on the trehalose concentration in the original solution. These results indicate that at concentrations below $20 \mathrm{wt} \%$, the size of each ice crystal is too large to be measured by TEM imaging, and very few ice crystals are present for TEM measurement. On the other hand, at concentrations above $20 \mathrm{wt} \%$, the size of the crystals decreases exponentially, reaching submicron orders of magnitude at $50 \mathrm{wt} \%$, whereas the number concentration increases exponentially. Therefore, it appears that the growth rate of the ice crystals in the high-concentration trehalose solution is reduced either by a reduction in the phase-separation rate between free water and the condensed solution or by a large supply of nucleation sites for ice crystals.

To confirm that the smooth surface corresponds to the ice crystal and to verify both size and number concentration data in 
lower concentration solutions, we observed a thin section of the trehalose solution, which was held between two glass plates and quenched in liquid nitrogen via a polarized optical microscope (POM) at approximately $258 \mathrm{~K}$. These data were shown as open triangles in Figs. 3 (a) and (b). The relationship between the average diameters and trehalose concentrations observed via the POM coincides well with hat observed via TEM. This is also the case with regard to the number density. Therefore, these figures support the assumption that the smooth surface observed using the TEM is the ice crystal grain, and that its size and number density showed an exponential dependence on the original trehalose concentration over the entire range of trehalose concentrations prepared in the present study.

As shown in Fig. 3a, we can control the size of the ice crystal formed in the quenched solution. The regression equation for the size of the ice crystal $\left(d_{\text {ice }}[\mu \mathrm{m}]\right)$ versus the trehalose concentration in the original solution $\left(C_{\text {treha }}[\mathrm{wt} \%]\right)$ is obtained as follows:

$$
d_{\text {ice }}=17.5 \exp \left[-6.34 \times 10^{-2} C_{\text {treha }}\right] \quad(\mathrm{R} \sim 0.96)
$$

Since the wavelength of visible light ranges between 0.36 and $0.83, d_{i c e}$ would be less than this range for the frozen solution at $C_{\text {treha }}>$ approximately $60 \mathrm{wt} \%$, which indicates that the ice crystal would be transparent. When we observe this phenomenon macroscopically, we might consider that the solution transforms into the glass phase.

We then observed the fine particle distribution in the intermediate spaces of the ice crystals. In some expanded observations, these particles were spherical or oval, with sizes ranging from several tens to a hundred nanometers in the same sample. In order to estimate the particle size distributions quantitatively, the diameter of the particles was expressed in terms of the diameter of a sphere of equivalent volume that was obtained by using either a spherical or a cylindrical approximation. The 
number density of the particles, which is normalized by the total number of particles (more than 50 particles) and the diameter ranges in intervals of $20 \mathrm{~nm}$ (less than $10 \mathrm{~nm}, 10-30 \mathrm{~nm}, 30-50 \mathrm{~nm}$, etc.), is shown in Fig. 4 (in which only six data points are shown). This figure shows that each distribution is well fitted to the log-normal distribution (shown by the solid line and various broken lines). Then, the mean diameter $\left(d_{p}\right)$ and standard deviations $\left(\sigma_{p}\right)$ of the particles in each sample were calculated by fitting the log-normal distribution. The number density of the fine particles $\left(N_{p}\right)$ was determined by the total number of particles in the observed area. We measured the number density from several different areas (more than five) in the same sample and averaged the number density values to observe the effect of the trehalose concentration.

Figure $5 \mathrm{a}$ and $\mathrm{b}$ show the variations in the $d_{p}$ and $N_{p}$ of the fine particles, respectively, with respect to the trehalose concentration in the original solution. Figure 5a shows that although there were several variations in terms of the size distributions, the $d_{p}$ value of each sample exhibited little dependence on the trehalose concentration. However, $N_{p}$ values of the fine particles were found to increase exponentially with an increase in the trehalose concentration (see Fig. 5b). The error bar shows the maximum variations of the measured $N_{p}$ values in the same sample. Note here that the gradual saturation of the number density in the samples with larger trehalose concentrations is considered to primarily result from the existence of ice crystals; that is because the number density measured in Fig. 2b (as a no ice-forming sample) is one order of magnitude larger than that shown in Fig. $5 \mathrm{~b}$ (as an open square). The total number of fine particles (estimated as $N_{p}$ $\left.\times \pi d_{p}{ }^{3} / 6\right)$ increases with the trehalose concentration. Therefore, it was observed that fine particles of almost the same size were distributed in the intermediate space of the ice crystals and that their area of occupation increased with the original trehalose concentration by an increase in the number density of the particles. These results support the assumption that the fine particles were related to trehalose molecules. 
It is well known that an unfrozen phase appears during the freezing of disaccharide solutions. Some previous studies on the freezing of sugar solutions $[12,13]$ have indicated that the concentration of sugars in this unfrozen phase is independent of the sugar concentration in the original solution. The volume of the unfrozen phase is therefore expected to increase with the sugar concentration in the solution. This phenomenon qualitatively coincides with the results observed in the present study, i.e., the area in which fine particles were found to increase with the trehalose concentration in the original solution. These results therefore suggest that the fine particles are precipitations of trehalose molecules from the unfrozen phase, and the remaining portion of the intermediate region of the ice crystals is in the glass phase of the solution.

Since these results are obtained from morphological observations on the replica sample, it is impossible to obtain any direct evidence for each phase. However, as shown previously, the ice crystals were identified with the help of POM observations. Here, we observed the variation in fine particle distributions with the extent of sample annealing at high temperatures to obtain indirect evidences for phases other than the ice crystal. We prepared three frozen samples of $35 \mathrm{wt} \%$ trehalose solution using the previously mentioned procedure. Then, Sample (A) was fractured at a temperature of approximately $90 \mathrm{~K}$ to obtain the reference sample. Sample (B) was stored in a vacuum chamber and heated until the sample temperature increased to approximately $180 \mathrm{~K}$. After approximately one hour, the sample was fractured to form the replica. Sample (C) was heated to approximately $250 \mathrm{~K}$ - sufficiently higher than the glass transition temperature of trehalose. Figure 6 shows the comparison of the TEM images for these three samples. The bar shows a length of $500 \mathrm{~nm}$, and all pictures were taken at the same magnification. Figure 6a verified that the obtained TEM image was the same as that shown in Fig. 1d. However, in samples (B) and (C), we found that most of the fine particles disappeared, and spherical or elongated flat surface areas were 
observed (Figs. 6b and 6c). The edge of the flat surface area in the sample (C) has a slightly larger contrast than that in sample (B). A comparison of these images may suggest that the fine particles disappeared due to higher temperature annealing. If the fine particles represent the trehalose precipitation and the surrounding part represents the glass phase of the solution with high trehalose concentration, this annealing effect can be explained by the melting of the precipitated fine particles to form the glass phase. At the highest temperature, the glass phase with high trehalose concentration would melt so as to form a droplet, which would result in a deeper hole on the fractured surface. Then, we concluded that the fine particles observed in Fig. 1 are the precipitations of trehalose molecules, and the remaining part consists of ice crystals and the glass phase.

Based on the conclusion that the fine particles represent the precipitation of trehalose molecules, we can qualitatively explain the inhibition mechanism of trehalose on ice crystal growth as follows. In a solution, trehalose molecules are hydrated (the hydration number is approximately 10 [13]). The hydrated $\mathrm{H}_{2} \mathrm{O}$ molecules are considered to be unable to contribute to ice formation. Then, if the trehalose concentration increases, the hydrated $\mathrm{H}_{2} \mathrm{O}$ in the solution would increase; as a result, the number of free $\mathrm{H}_{2} \mathrm{O}$ molecules would decrease. When a solution is frozen under such conditions, the volume of the unfrozen phase would increase, whereas that of the ice crystals would decrease. The increase in the volume of the unfrozen phase would then result in the separation of the free $\mathrm{H}_{2} \mathrm{O}$ phase into small areas. If nucleation occurs easily and homogeneously in the entire solution, the size of each ice crystal grain would decrease. These processes are fairly similar to those predicted based on the basis of molecular dynamics simulations [12] and those estimated from the experimental measurements of the freezing points of sugars [3]. If the ice crystals are sufficiently smaller than living cells, then the mechanical damage caused by the ice crystals to the living cells would be reduced during the process of freezing the 
solution. Since the growth rate of the ice crystal surrounded by the high-concentration trehalose solution is small, the morphology of each ice crystal would be granular and spherical. This may also result in the reduction of the mechanical damage caused by the ice crystals to the living cells during freezing. The reduction effect of the size of the ice crystals may also be effective for the use of trehalose as a cryoprotectant.

\section{<Formation process of fine particles during freezing $>$}

As regards the distribution of fine particles, Figure 1 shows that the fine particles are distributed in a relatively homogeneous manner at the boundary between the ice crystals in the samples with higher trehalose concentrations (e.g.,

Figs. 1e and f), whereas the fine particles are not homogeneously distributed in the samples at lower concentrations (e.g., Figs. 1a-d). The figure also indicates that the formation of fine particles is easier than growth; however, they would agglomerate after formation. We will now address the process of the formation of fine particles in more detail based on the examination of various TEM images.

As discussed previously, each fine particle is considered to consist of trehalose molecules. Based on the knowledge that the disaccharide concentration in the unfrozen phase is independent of that in the original solution [12, 13], the qualitative formation process of fine particles during freezing would be as follows. When the solution is cooled below the freezing point, ice crystals start to form. Since ice crystals tend to exclude solutes from the solid phase, the trehalose concentration in the remaining unfrozen phase increases. This process represents the fractionation between the trehalose solution and free water, and will continue until the trehalose concentration reaches a critical value. This critical concentration would coincide 
with the concentration observed in the glass phase $C_{g}$ ' $[12,13]$, which is estimated to be marginally higher than the maximum trehalose concentration in the original solution-approximately $65 \mathrm{wt} \%$ or $0.084 \mathrm{~mol} \mathrm{~mol}^{-1}$. When the concentration exceeds $\mathrm{C}_{\mathrm{g}}$ ' during freezing, the trehalose molecules would start to precipitate and form fine particles. Based on the microscopic observations, a large number of uniform-sized fine particles are formed. Therefore, we consider that the formation of fine particles is easier than their growth; however, they would agglomerate after the formation. This precipitation may maintain a constant trehalose concentration in the unfrozen phase.

As shown in the particle-size histograms (Fig. 4), the minimum particle size is at most $10 \mathrm{~nm}$. We estimated the number of trehalose molecules in the smallest particles in order to observe the particle-precipitate processes in the solution. The specific volume of one trehalose molecule was estimated to be approximately $0.415 \mathrm{~nm}^{3}$ on the basis of X-ray diffraction analysis [14]. However, in the solution, the trehalose molecules were hydrated and slightly deformed with changes in the glycosidic dihedral angles [12]. Trehalose molecules are hydrated with approximately $10 \mathrm{H}_{2} \mathrm{O}$ molecules [13]. The specific volume of one hydrated trehalose molecule is approximately $0.8 \mathrm{~nm}^{3}$. If we assume that the minimum size of an agglomerated particle is a sphere with a diameter of less than $10 \mathrm{~nm}$, the number of hydrated trehalose molecules in the particle is approximately 500. Even if we consider any uncertainties included in the above estimations, it is feasible to assume that the smallest particles consist of several hundreds of trehalose molecules. Previous studies on trehalose solutions (e.g., [12]) have suggested that trehalose molecules tend to form clusters in a solution. It is difficult to quantitatively estimate the size of the clusters in the solution, but the results obtained in the present study allowed us to speculate that the cluster size is less than $10 \mathrm{~nm}$ in diameter, which would include several hundreds of trehalose molecules. 
Here, we note that the formation process of fine particles in the trehalose solution is very similar to the self-association process of colloidal particles. In general, the size of a colloidal particle ranges from several nanometers to submicrons. They appear in the solution in which the concentration exceeds the critical value. The aggregated particle size is almost constant, and the number of particles increases when the concentration increases. Then, we assume that the fine particles observed in the present study represent the associated colloid of trehalose molecules. The critical aggregation concentration $C_{C A C}$ of the colloid association is given by the following equation [15]:

$$
C_{C A C} \sim \exp \left[-4 \pi r^{2} \gamma / \mathrm{kT}\right]
$$

where $r$ is the molecular radius; $\gamma$, the interfacial free energy per unit area; $\mathrm{k}$, the Boltzmann constant; and $T$, the absolute temperature. Here, we consider that the association conditions of the colloid coincide with the glass-transition conditions of the trehalose solution, that is, $T=\mathrm{T}_{\mathrm{g}}{ }^{\prime}=230.2 \mathrm{~K}$ and $C_{C A C}=\mathrm{C}_{\mathrm{g}}{ }^{\prime}=0.084 \mathrm{~mol} \mathrm{~mol}^{-1}$ [13]. As discussed previously, $r$ is approximately $0.5 \mathrm{~nm}$. Then, we obtain the apparent interfacial free energy of trehalose aggregation in the solution to be approximately $\gamma_{p} \sim 2.5 \mathrm{~mJ} \mathrm{~m}^{-2}$. This value is one order smaller than the interfacial free energy between ice and water [16].

Since the minimum size of the particles appears to be constant and the formation of the particles is easier than their growth, we estimate how easily the fine particles are formed. On the basis of the homogeneous nucleation theory, the critical size of the spherical phase $r_{c}$ is obtained by the following equation.

$$
r_{c}=2 v \gamma_{P} \Delta \mu_{P}^{-1}
$$

where $v$ is the molar volume of trehalose and $\Delta \mu_{P}$ is the free energy difference between the dispersed and precipitated states of trehalose molecules in water. After substituting $v=4.8 \times 10^{-4} \mathrm{~m}^{3} \mathrm{~mol}^{-1}, \gamma_{p}=2.5 \mathrm{~mJ} \mathrm{~mol}^{-1}$, and $r_{c}=5 \times 10^{-9} \mathrm{~m}$, we obtain a $\Delta \mu_{P}$ value to be approximately $0.5 \mathrm{~kJ} \mathrm{~mol}^{-1}$. This value of $\Delta \mu_{P}$ is approximately one order smaller than that of the 
formation energy of ice crystals in water. This estimation may indicate that the interaction among the agglomerated trehalose molecules (clusters) is much weaker than that between the water and trehalose molecules. The trehalose clusters tend to disperse into the solution when the trehalose concentration is low, but they easily agglomerate when the concentration increases.

When the initial trehalose concentration is high, fine particles may precipitate prior to ice crystal formation due to the temperature decrease and the ease of precipitation as compared to ice nucleation. In such a case, the ice crystal would nucleate with the assistance of the fine particles, i.e., due to the presence of the interface as a nucleation site or due to thermal disturbance resulting from the heat of precipitation. Because the precipitation occurs homogeneously, the nucleation of ice crystals appears to occur spontaneously. This speculation could be supported by the fact that the number density of ice crystals formed in high-concentration solutions appeared to be extremely large and that the ice crystals exhibited relatively similar shapes and sizes (e.g., Figs. 1e and f). In addition, if the fine particles precipitated prior to the ice crystal formation, the growth rate of ice crystals in the high-concentration trehalose solution was slow due to the slow supply of $\mathrm{H}_{2} \mathrm{O}$ molecules from the surrounding solution. This interpretation is supported by the fact that many of the observed ice crystals had facets, which were usually observed at relatively slow crystal growth rates as compared to the freezing rate in the present study. In such cases, the growth of ice crystals would also be inhibited kinetically by the presence of fine particles.

\section{<Comparison of trehalose solution with other disaccharide solutions>}

In order to demonstrate the unique characteristics of the trehalose solution observed in the present study, we compared the 
TEM images of the replica samples formed with trehalose to those formed with two other disaccharides: sucrose and maltose. The details of the experimental procedures were similar to those used for the trehalose solution experiments. The freezing rate was fixed at $3 \times 10^{2} \mathrm{~K} \mathrm{~min}^{-1}$ for the comparisons. The prepared solutions of both the alternative disaccharides were within the same concentration range as that used for trehalose.

Figure 7 shows a representative image of a sucrose solution (35 wt\% concentration). We observed features in the sucrose solutions that were similar to those observed in the trehalose solutions: there were very smooth surfaces and fine particles distributed between the smooth surfaces. As discussed in the previous sections, the smooth areas were considered to be ice crystals, and the fine particles were considered to reflect the precipitation of sucrose molecules. The size of the fine particles exhibited a similar range to that in trehalose. However, the fine particles appeared to agglomerate to form another type of flat surface (e.g., the lower point of Fig. 7). The fine particles tended to localize near the boundary between the ice crystals and the sucrose-agglomerated area. This may represent the quenched feature of fine-particle precipitation. We speculate that the sucrose molecules precipitated when the ice crystals began to grow. In other parts, we sometimes observed that the fine particles were located at the edge of the ice crystal grain. This may have been caused because the precipitated fine particles were collected by the migration of the boundary between the ice crystal and the surrounding glass phase that included the fine particles. Since the number density of the fine particles was smaller than that of trehalose, we consider that the energy barrier of sucrose precipitation would be higher than that of trehalose.

On the other hand, because the ice crystals were still too large for observation of their gross features by TEM, even at the highest sucrose concentration (50 wt\%), we were unable to estimate the size changes using the changes in the sucrose 
concentration. Thus, the inhibitory effect of sucrose on ice crystal growth is weaker than that of trehalose.

The freeze-fractured features of maltose solutions differed from those observed in either trehalose or sucrose solutions. Figure 8 shows a representative image of a maltose solution (35 wt\% concentration). We observed smooth areas with some boundaries; however, only a small number of fine particles were observed, even in the sample from the $50 \mathrm{wt} \%$ solution. As shown in Fig. 8, some fine particles were observed at the edge of the smooth area. Their size was almost the same as that of the particles observed in the other disaccharide solutions. Comparing the number densities of fine particles in the same concentration of disaccharide (e.g., 35 wt\%), we can consider that maltose might be the most difficult solute for fine particle precipitation during the cooling. These particles were considered to be collected there by boundary migration. Since the curvature of the grain boundary was relatively small, it is likely that the maltose-condensed phase might have reduced the growth rate of the ice crystals to a certain extent. However, similar to the case of the sucrose solutions, we were unable to estimate the precise size of the ice crystals, because they were still too large for the TEM observation of the gross features of individual ice crystals. Therefore, we concluded that the maltose molecules were relatively more difficult to precipitate in an unfrozen solution than other disaccharides and that maltose molecules have a weaker effect on the inhibition of ice crystal growth than other disaccharide molecules.

As regards the hydration of disaccharides, Furuki and Sakurai [13] noted that trehalose has the largest hydration number, followed by maltose and lastly, sucrose. The difference between the hydration numbers of trehalose and sucrose was approximately $15 \%$. However, based on the observation in our present study, the difference in the inhibitory effect of ice crystal growth between these disaccharides is greater than that expected from the hydration number difference. Molecular 
dynamics simulations [12] suggested that the values of certain properties of the maltose solution were almost directly between those of the trehalose and sucrose solutions. Then, it may be reasonable to assume qualitatively that trehalose plays the most effective role in the inhibition of ice crystal growth due to the reduction of free water molecules from the unfrozen solution.

However, it remains difficult to represent the inhibitory effects of trehalose quantitatively based only on the fact that it has the largest hydration number of the three disaccharides. In the present study, we found that the disaccharides tend to precipitate in high-concentration solutions, which might affect the inhibition of ice crystal growth. Thus, the pinning effect of the precipitated fine particles during ice crystal growth would be another process of inhibition. It is noteworthy in this context that trehalose plays another important role. Trehalose-a nucleation promoter-enhances the nucleation of ice crystals, which results in the inhibition of the growth of individual ice crystals. These modes of kinetic inhibition are expected to be important for gaining a better understanding of the precise effects of disaccharides on the inhibition of ice crystal growth.

Since trehalose and sucrose are utilized in living organisms that are capable of enduring cold temperatures, whereas maltose is not, the conclusive role of disaccharides as cryoprotectants would be not only to inhibit ice crystal growth, but also to protect the cell membrane. Some previous simulation studies (e.g., $[2,17])$ have suggested that disaccharide molecules tend to stabilize the structure of the phospholipids bilayer since they bridge adjacent lipid head groups and preserve the membrane under cooling conditions without affecting its structure. The precipitation phenomenon renders this process effective due to the enhancement of the contact between the cell membrane and a large concentration of disaccharide 
molecules. Further experiments and theoretical analyses will be necessary to reveal the actual mechanisms involved in the cryoprotection of living organisms.

\section{Conclusions}

We observed replica samples of a freeze-fractured trehalose solution via FE-TEM to reveal the mechanism of ice crystal growth inhibition. Quenched ice samples prepared from trehalose solutions of various concentrations ranging from $3 \mathrm{wt} \%$ to $50 \mathrm{wt} \%$ were fractured in a vacuum chamber, and the platinum-carbon coating on the fresh surface allowed us to observe the nanometer-scale features of each sample.

The TEM images revealed that there were two distinct areas: a smooth surface and an area that included homogeneously distributed fine particles (with an average diameter of several tens of nanometers). A comparison between the various images of a variety of samples prepared at different trehalose concentrations and different freezing rates led to the conclusion that the smooth surface represents the ice crystal, and the fine particles reflect the precipitation phase of the trehalose molecules. The remaining area around the fine particles might be the glass phase of the trehalose solution.

The minimum size of the fine particle, which consists of several hundreds of trehalose molecules, was estimated to be approximately $10 \mathrm{~nm}$ in diameter and was considered to originally exist in the solution as a trehalose-agglomerated cluster. Trehalose molecules would easily form large clusters in the solution, and these molecules could easily precipitate from the solution when the trehalose concentration exceeded a certain critical value during its cooling. However, a confirmation 
study will be required to verify that the fine particle is the precipitation of disaccharide molecules.

A careful observation of the fine particle distributions and ice crystal features led to the conclusion that the primary mode of inhibition of ice crystal growth in trehalose solution is the decrease in the supply of free water molecules from the unfrozen solution to that of the ice crystal phase. This process is primarily due to the strong interaction between the trehalose and water molecules. In order to further examine these conclusions, we observed the replica samples of the other disaccharides—sucrose and maltose—during the same experimental procedures. A comparison of these TEM images with those of trehalose suggested that the fine particles in the case of sucrose and maltose tended to reduce the rate of ice crystal growth; however, the inhibitory effect of trehalose on ice crystal growth was the greatest among these disaccharides. The primary reason for the relative effectiveness of trehalose on the inhibition of ice crystal growth was that trehalose plays not only a static role (i.e., the reduction in the supply of free water molecules) but also various dynamic roles (such as the pinning effect of precipitated fine particles on the step mobility of the ice crystal and the enhancement of ice nucleation during freezing).

\section{Acknowledgements}

Trehalose and maltose were donated by Dr. H. Chaen and Dr. M. Kubota (Hayashibara Biochemical Labs., Inc.). The authors gratefully acknowledge Mr. A. Okutomi for his technical support in the preparation of the replica samples, and Dr. N. Sakaguchi (Hokkaido Univ.) and Mr. H. Kurotaki (JEOL) for their support in the TEM observations. The authors would also like to thank Professor T. Sei (Aichi Gakuen Univ.), Professor S. Fujikawa (Hokkaido Univ.), Dr. K. Akao (JASCO), 
and Professor Y. Tominaga (Ochanomizu Univ.) for fruitful discussions regarding the study. Experimental operations in the cold room were supported by Dr. A. Miyamoto, Dr. S. Horikawa and Professor T. Hondoh (Hokkaido Univ.). 


\section{References}

[1] Crowe , J. H., L. M. Crowe, J. F. Carpenter, A. S. Rudolph, C. A. Wistrom, B. J. Spargo and T. J., Anchrodoguy, Interactions of sugars with membranes. Biochim. Biophys. Acta. 947 (1988) 367-384.

[2] Sum, A. K., R. Faller and J. J. de Pablo, Molecular simulation study of phospholipids bilayers and insights of the interactions with disaccharides, Biophys. J. 85 (2003) 2830-2844.

[3] Sei, K., T. Gonda and Y. Arima, Freezing of the solution of trehalose and water (in Japanese with English abstract), Cryobio. Cryotech., 47 (2001) 9-12.

[4] Sei, K., T. Gonda and Y. Arima, Growth rate and morphology of ice crystals growing in a solution of trehalose and water, J. Crystal Growth, 240 (2002) 218-229.

[5] Sei, K. and T. Gonda, Melting points of ice crystals growing in sugar solutions (in Japanese with English abstract), Cryobio. Cryotech., 50 (2004) 93-95.

[6] Wang, Y. and Y. Tominaga, Dynamical structure of water in aqueous solutions of D-glucose and D-galactose by low-frequency Raman scattering, J. Chem. Phys. 100 (1994) 2407-2412.

[7] Kanno, H. and Y. Yamazaki, Raman study of aqueous solutions of disaccharides including trehalose (in Japanese with English abstract),. Cryobio. Cryotech., 47 (2001) 76-79.

[8] Akao, K., Y. Okubo, H. Masago, N. Asakawa, Y. Inoue and M. Sakurai, FTIR study of the properties of anhydrous form II of trehalose (in Japanese with English abstract), Cryobio. Cryotech., 47 (2001) 23-26.

[9] Branca, C., S. Magazu, G. Maisano and P. Migliardo, Anomalous cryoprotective effectiveness of trehalose: Raman scattering evidences, J. Chem. Phys., 111 (1999) 281-287. 
[10] Franks, F., M. H. Asquith, H. leB. Skare and B. Roberts, Aggregation patterns and microstructure in aqueous polymer solutions: - comparison of quench-fracture electron microscopy with predictions based on rheological measurements, Cryo-Lett. 1 (1979) 104-113.

[11] Levin, H., L. Slade, Principles of “cryostabilization” technology from structure/property relationships of carbohydrate/water systems - A review, Cryo-Lett., 9 (1988) 21-63.

[12] Lerbret, A., P. Bordat, F. Affouard, M. Descamps and F. Migliardo, How homogeneous are the trehalose, maltose, and sucrose water solutions? An insight from molecular dynamics simulations, J. Phys. Chem. B, 109 (2005) 11046-11057.

[13] Furuki, T., Effect of molecular structure on thermodynamic properties of carbohydrates. A calorimetric study of aqueous di- and oligosaccharides at subzero temperatures, Carbohydrate Res., 337 (2002) 441-450; Furuki, T., M. Sakurai, Bioprotective mechanism of a, a-trehalose in view of hydration property and phase transition behavior (in Japanese with English abstract), Cryobio. Cryotech., 51 (2005) 1-11.

[14] Taga, T., M. Senma, K. and Osaki, The crystal and molecular structure of trehalose dehydrate, Acta Cryst., B28 (1972) $3258-3263$.

[15] Israelachvili, J. N., Intermolecular and surface forces $2^{\text {nd }}$ ed., Academic Press Ltd., London, (1992) pp. 450.

[16] Fletcher, N. H., The Chemical Physics of Ice, Cambridge Univ. Press., London, (1970) pp. 271.

[17] Lee, B. W., R. Faller, A. K. Sum, I. Vattulainen, M. Patra and M. Karttunen, Structure effects of small molecules on phospholipids bilayers investigated by molecular simulations, Fluid Phase Equilibria, 225 (2004) 63-68. 
Figure Captions

Fig. 1a: TEM image of an ice surface formed from trehalose solution (8.5 wt\%). Scale bar: $100 \mathrm{~nm}$.

Fig. 1b: TEM image of an ice surface formed from trehalose solution (15 wt\%). Scale bar: $100 \mathrm{~nm}$.

Fig. 1c: TEM image of an ice surface formed from trehalose solution (25 wt\%). Scale bar: $100 \mathrm{~nm}$. The inset picture was taken at a smaller magnification (scale bar: $500 \mathrm{~nm}$ ).

Fig. 1d: TEM image of an ice surface formed from trehalose solution (35 wt\%). Scale bar: $100 \mathrm{~nm}$.

Fig. 1e: TEM image of an ice surface formed from trehalose solution (40 wt\%). Scale bar: $100 \mathrm{~nm}$. The inset picture was taken at a smaller magnification (scale bar: $200 \mathrm{~nm}$ ).

Fig. 1f: TEM image of an ice surface formed from trehalose solution (50 wt\%). Scale bar: $100 \mathrm{~nm}$.

Fig. 2a: TEM image of an ice surface formed from trehalose solution (40 wt\%, slow). Scale bar: $100 \mathrm{~nm}$.

Fig. 2b: TEM image of an ice surface formed from trehalose solution (35 wt\%, rapid). Scale bar: $100 \mathrm{~nm}$.

Fig. 3a: Trehalose concentration dependence on the average diameter of ice crystals. Solid circles were observed via TEM and open triangles were via POM.

Fig. 3b: Trehalose concentration dependence on the number density of ice crystals. Solid circles were observed via TEM and open triangles were observed via POM.

Fig. 4: Histogram of the number density ratio of fine particles in various trehalose concentrations. Each histogram was fitted to the log-normal distribution functions denoted by solid and broken lines.

Fig. 5a: Trehalose concentration dependence on the average diameter of fine particles $\left(d_{p}\right)$. Open circles are estimated by fitting the log-normal distribution functions. The error bars show the standard deviations $\left(\sigma_{p}\right)$ obtained by the curve 
fitting in Fig. 4. Solid circles are estimated by the arithmetic mean value because of the limited number of particles. The error bars for them denote the minimum and maximum diameter observed in the same sample.

Fig. 5b: Trehalose concentration dependence on the number density of fine particles $\left(N_{p}\right)$. The open square denotes the average number density of the fine particles observed in Fig. 2b.

Fig. 6: TEM images of the ice surface formed from trehalose solution (35 wt\%) fractured at temperatures (a) approximately 90 K, (b) approximately $180 \mathrm{~K}$, and (c) approximately $250 \mathrm{~K}$. Scale bar: $500 \mathrm{~nm}$.

Fig. 7: TEM image of an ice surface formed from sucrose solution (35 wt\%). Scale bar: $100 \mathrm{~nm}$.

Fig. 8: TEM image of an ice surface formed from maltose solution (35 wt\%). Scale bar: $100 \mathrm{~nm}$. 
Uchida et al. Fig. 1a
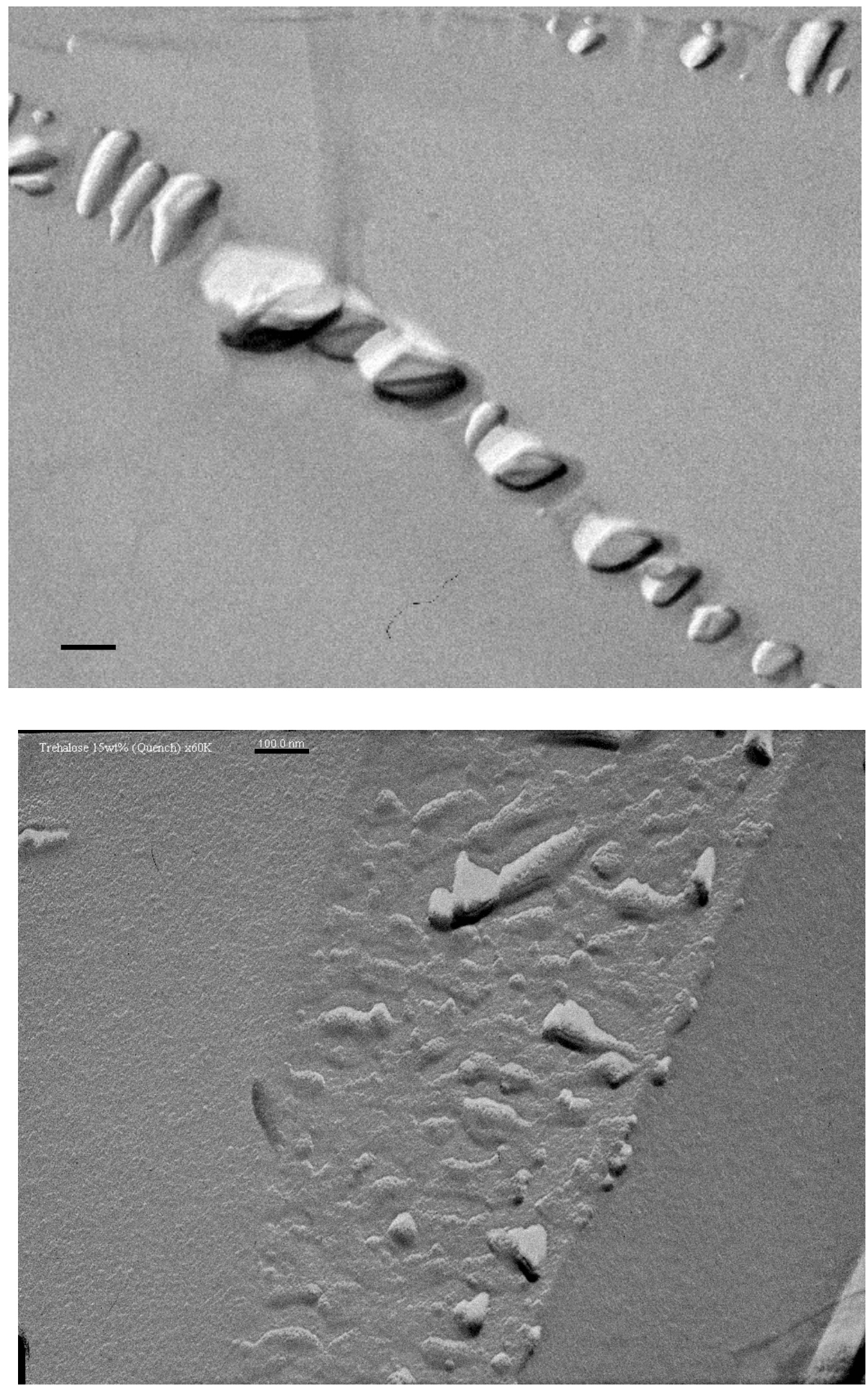

Uchida et al. Fig. 1b 


\section{Uchida et al. Fig. 1c}
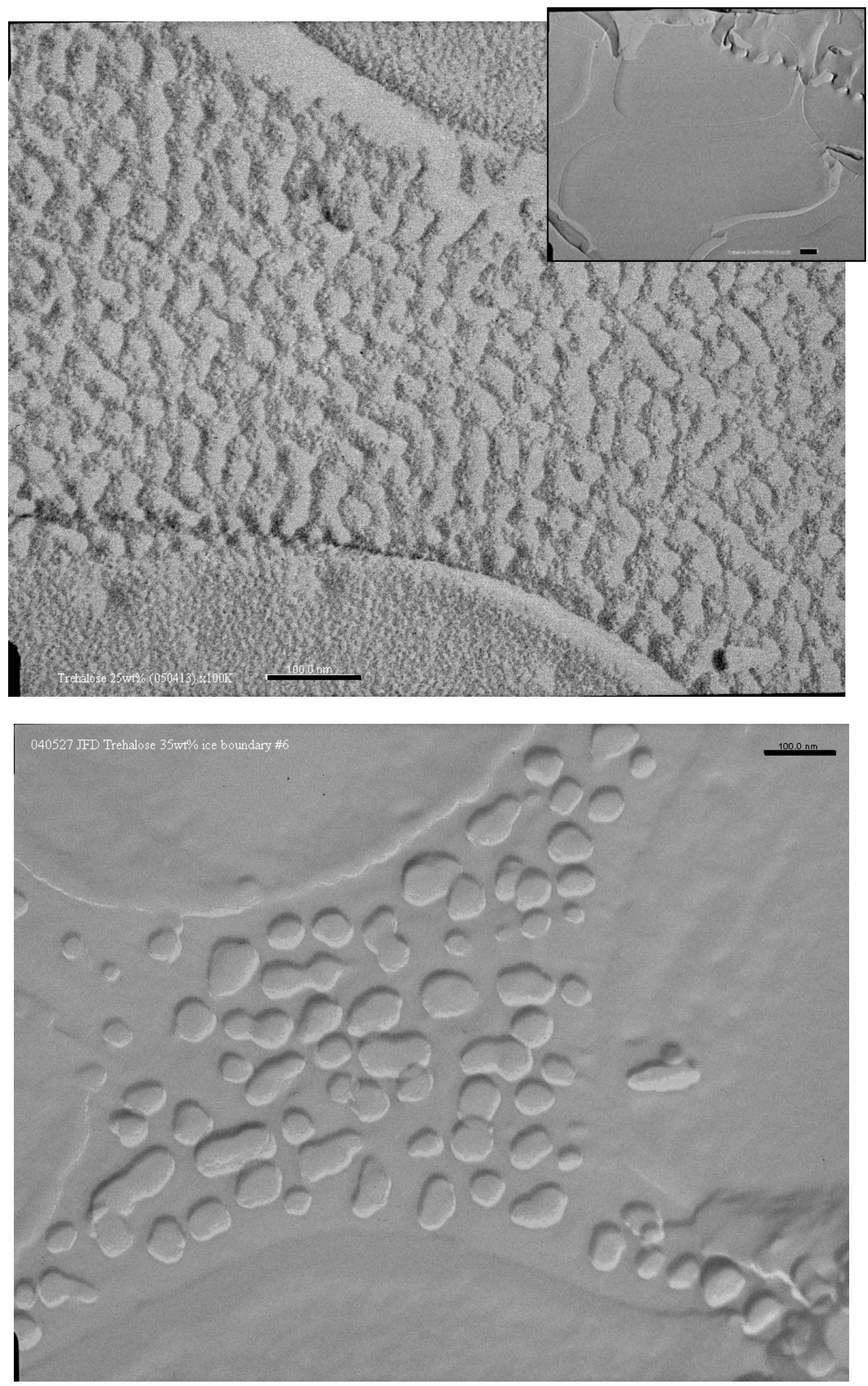

Uchida et al. Fig. 1d 
Uchida et al. Fig. 1e
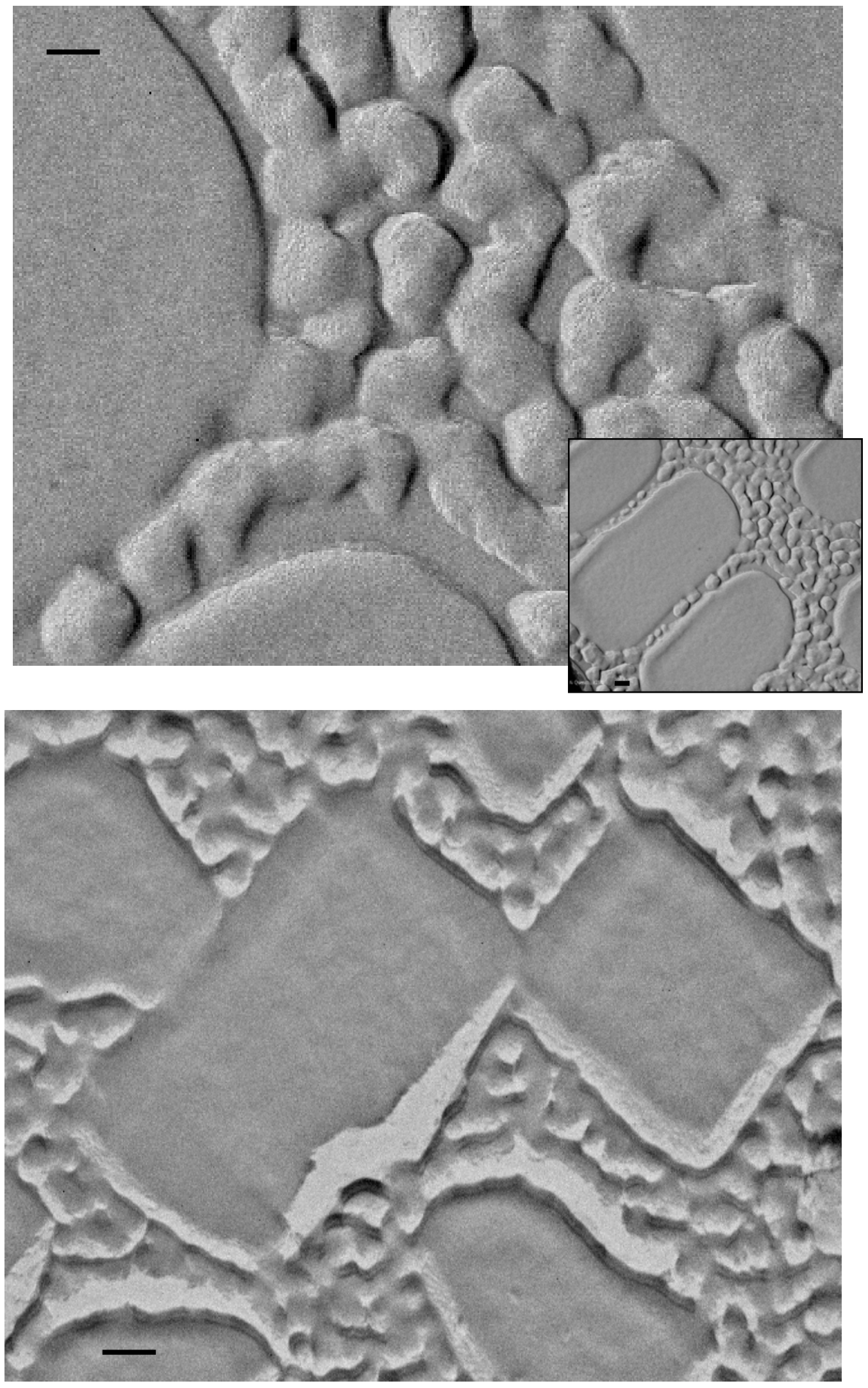

Uchida et al. Fig. If 
Uchida et al. Fig. 2a
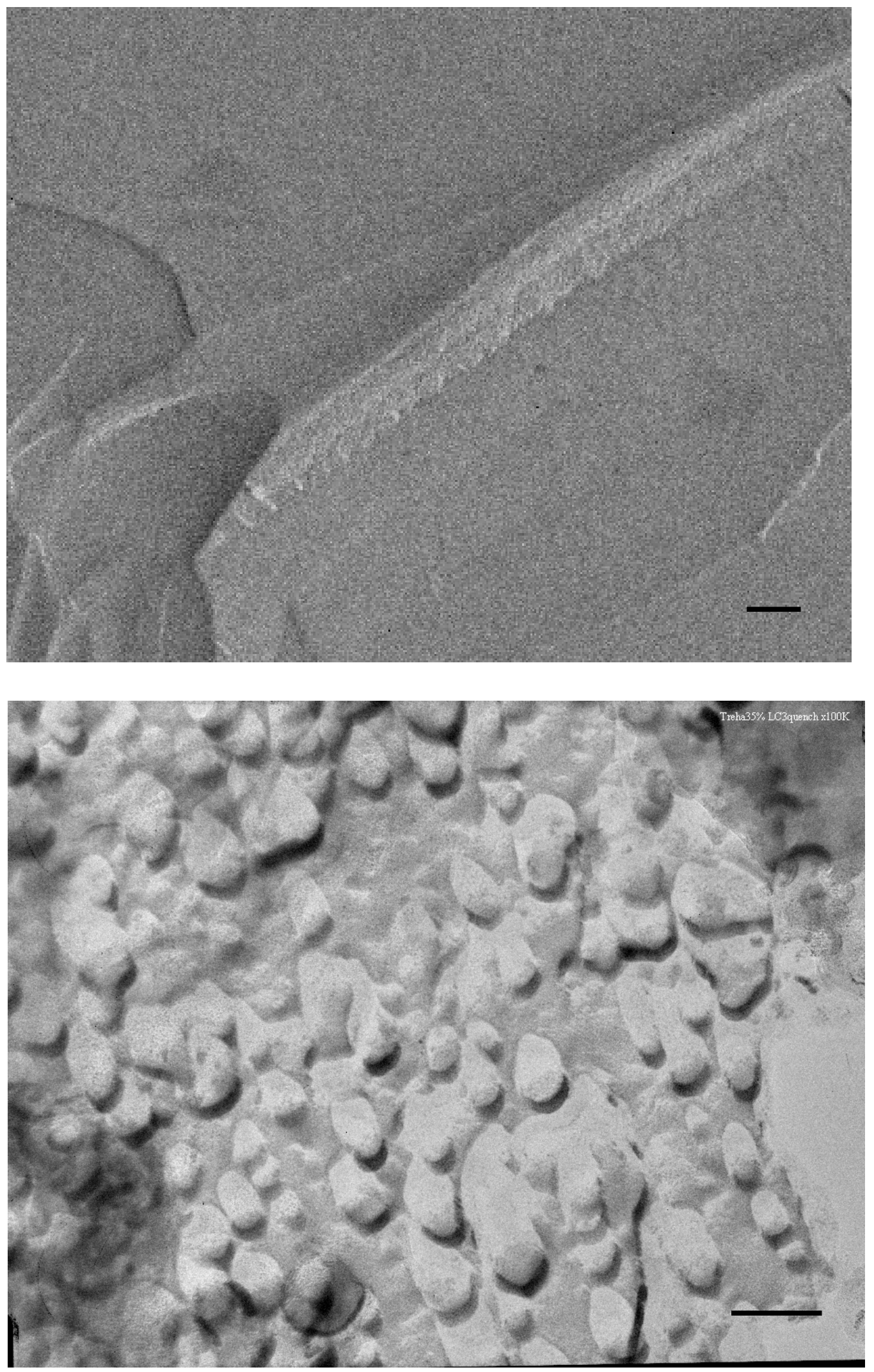

Uchida et al. Fig. 2b 
Uchida et al. Fig. 3a
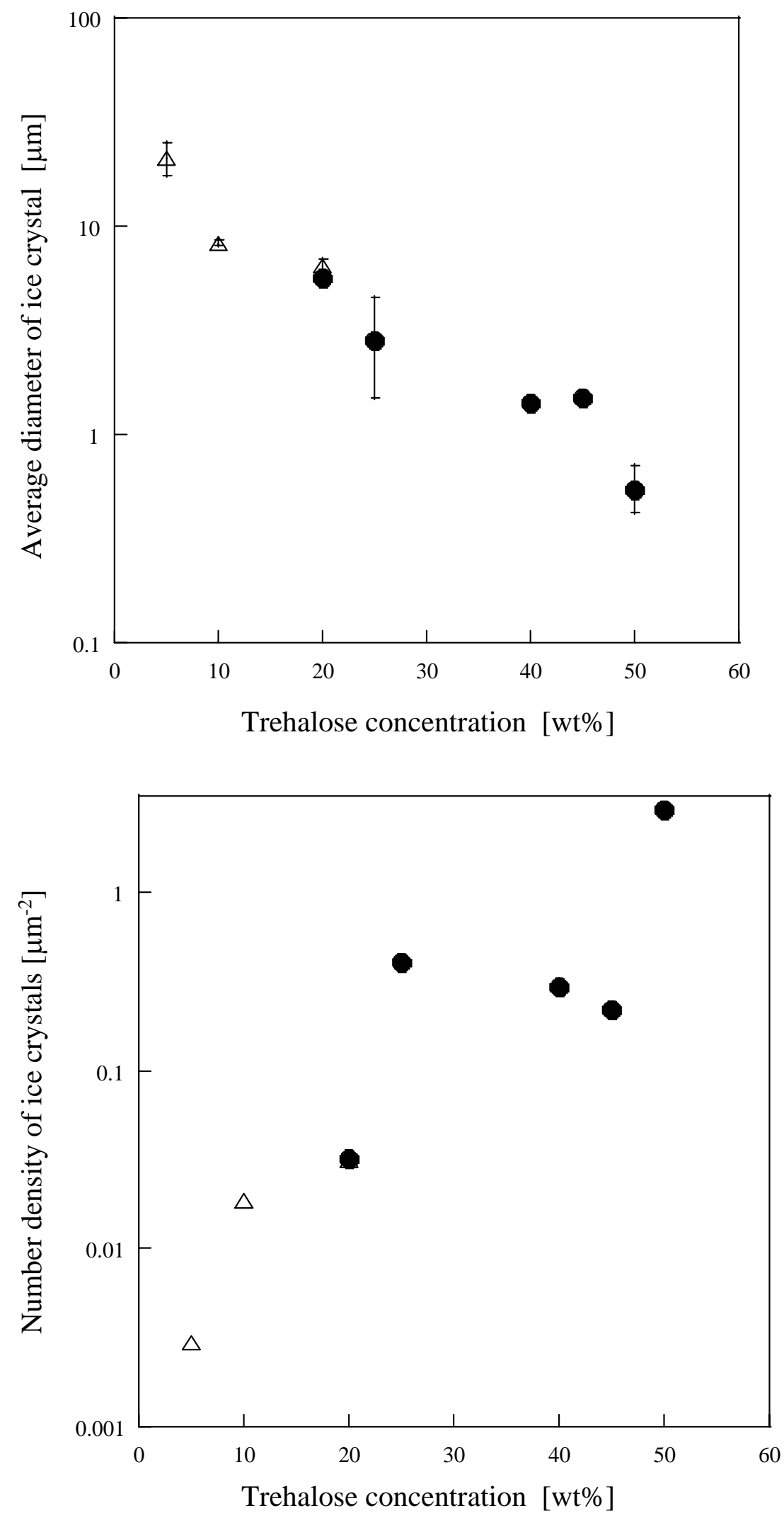

Uchida et al. Fig. 3b 


\section{Uchida et al. Fig. 4}

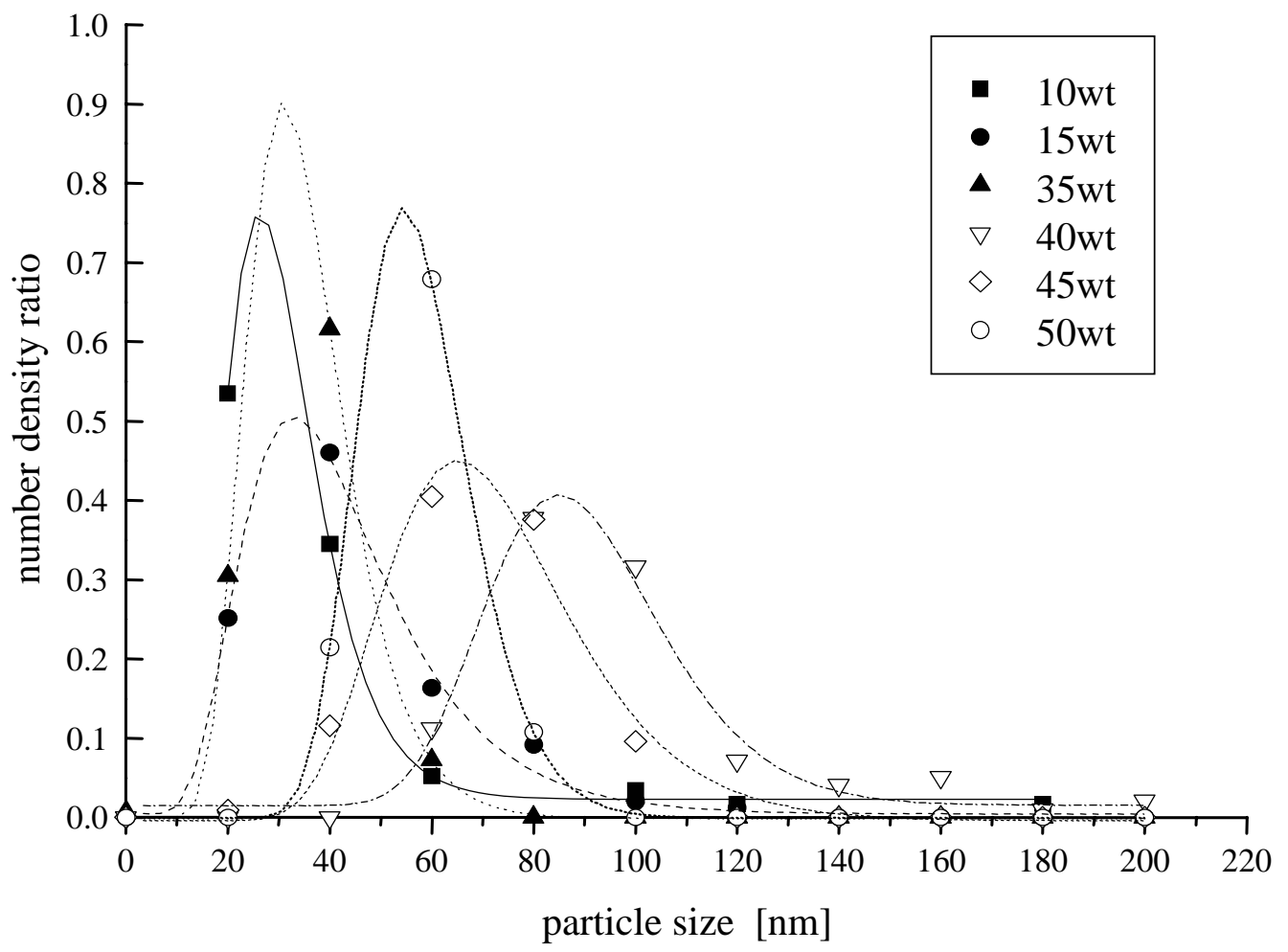


Uchida et al. Fig. 5a
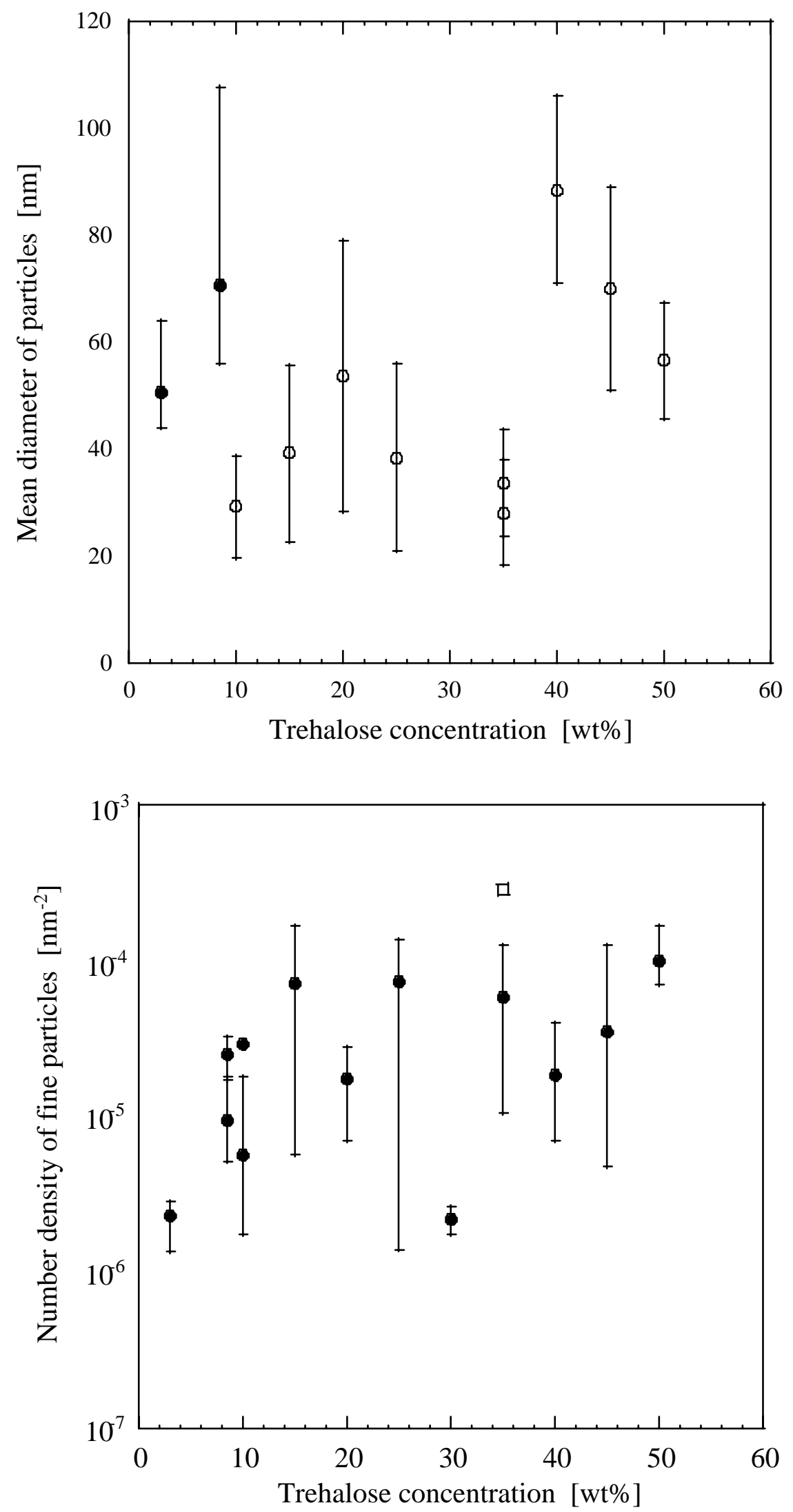

Uchida et al. Fig. 5b 
Uchida et al. Fig. 6

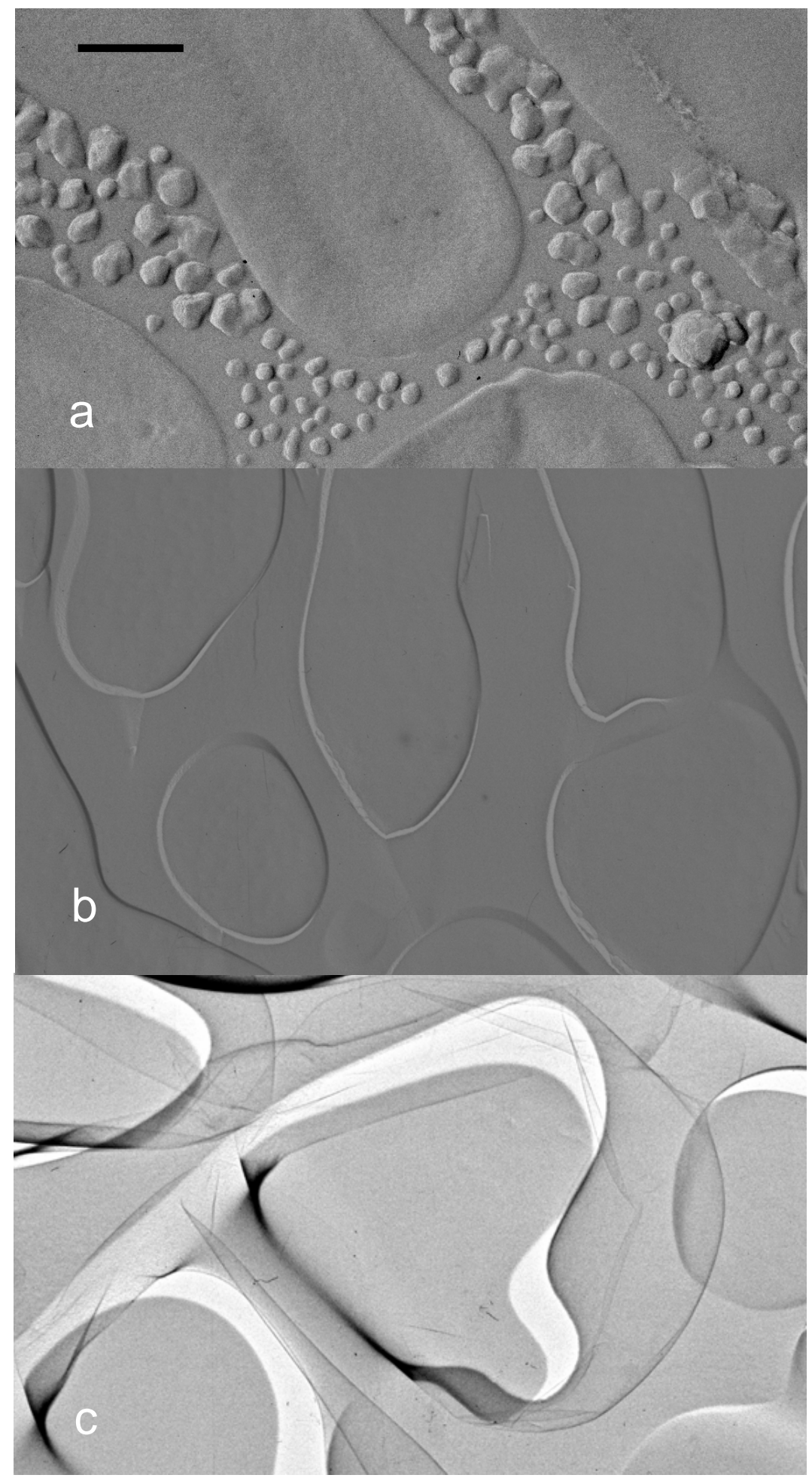


Uchida et al. Fig. 7
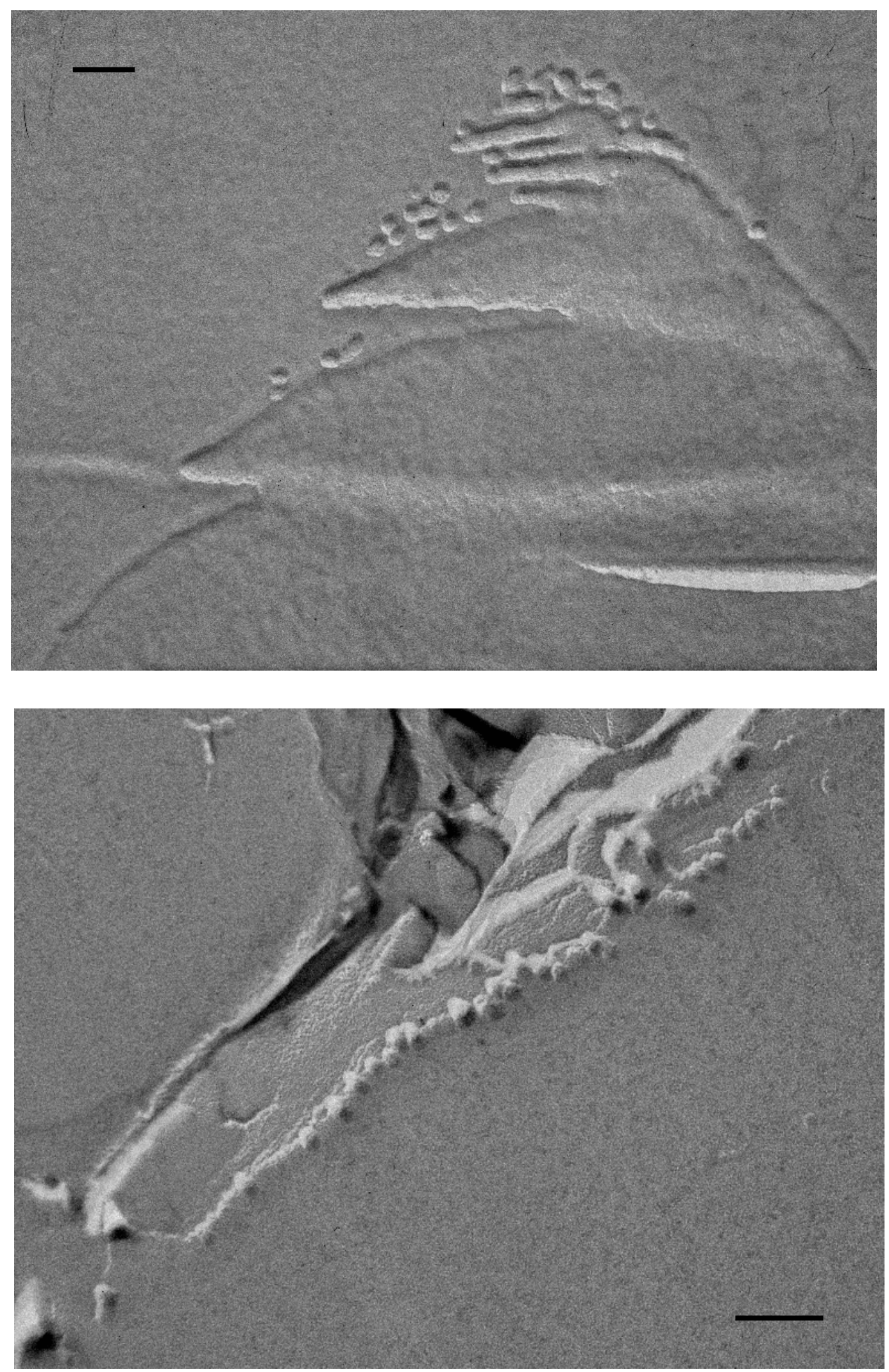

Uchida et al. Fig. 8 